\title{
ON SOME PERMUTATION POLYNOMIALS OVER FINITE FIELDS
}

\author{
AMIR AKBARY AND QIANG WANG
}

Received 8 March 2005 and in revised form 5 May 2005

Let $p$ be prime, $q=p^{m}$, and $q-1=7 s$. We completely describe the permutation behavior of the binomial $P(x)=x^{r}\left(1+x^{e s}\right)(1 \leq e \leq 6)$ over a finite field $\mathbb{F}_{q}$ in terms of the sequence $\left\{a_{n}\right\}$ defined by the recurrence relation $a_{n}=a_{n-1}+2 a_{n-2}-a_{n-3}(n \geq 3)$ with initial values $a_{0}=3, a_{1}=1$, and $a_{2}=5$.

\section{Introduction}

Let $\mathbb{F}_{q}$ be a finite field of $q=p^{m}$ elements with characteristic $p$. A polynomial $P(x) \in$ $\mathbb{F}_{q}[x]$ is called a permutation polynomial of $\mathbb{F}_{q}$ if $P(x)$ induces a bijective map from $\mathbb{F}_{q}$ to itself. In general, finding classes of permutation polynomials of $\mathbb{F}_{q}$ is a difficult problem (see [3, Chapter 7] for a survey of some known classes). An important class of permutation polynomials consists of permutation polynomials of the form $P(x)=x^{r} f\left(x^{(q-1) / l}\right)$, where $l$ is a positive divisor of $q-1$ and $f(x) \in \mathbb{F}_{q}[x]$. These polynomials were first studied by Rogers and Dickson for the case $f(x)=g(x)^{l}$, where $g(x) \in \mathbb{F}_{q}[x][3$, Theorem 7.10]. A very general result regarding these polynomials is given in [8]. In recent years, several authors have considered the case that $f(x)$ is a binomial (e.g., [2, 9] and [1]).

Here we consider the binomial $P(x)=x^{r}+x^{u}$ with $r<u$. Let $s=(u-r, q-1)$ and $l=$ $(q-1) / s$. Then we can rewrite $P(x)$ as $P(x)=x^{r}\left(1+x^{e s}\right)$, where $s=(q-1) / l$ and $(e, l)=1$. If $P(x)=x^{r}\left(1+x^{e s}\right)$ is a permutation binomial of $\mathbb{F}_{q}$, then $P(x)$ has exactly one root in $\mathbb{F}_{q}$ and thus $l$ is odd. When $l=3,5$, the permutation behavior of $P(x)$ was studied by Wang [9]. In the case $l=5$, the permutation binomial $P(x)$ is determined in terms of the Lucas sequence $\left\{L_{n}\right\}$, where

$$
L_{n}=\left(2 \cos \frac{\pi}{5}\right)^{n}+\left(-2 \cos \frac{2 \pi}{5}\right)^{n}
$$

More precisely, it is proved that under certain conditions on $r, s=(q-1) / 5$, and $e$, the binomial $P(x)=x^{r}\left(1+x^{e s}\right)$ is a permutation binomial if and only if $L_{s}=2$ in $\mathbb{F}_{p}[9$, Theorem 2]. 
In this paper, we consider the case $l=7$ (see [1] for some results related to general $l$ ). Here we introduce a Lucas-type sequence $\left\{a_{n}\right\}$ by

$$
a_{n}=\left(2 \cos \frac{\pi}{7}\right)^{n}+\left(-2 \cos \frac{2 \pi}{7}\right)^{n}+\left(2 \cos \frac{3 \pi}{7}\right)^{n}
$$

for integer $n \geq 0$. It turns out that $\left\{a_{n}\right\}_{n=0}^{\infty}$ is an integer sequence satisfying the recurrence relation

$$
a_{n}=a_{n-1}+2 a_{n-2}-a_{n-3}
$$

with initial values $a_{0}=3, a_{1}=1$, and $a_{2}=5$ (see Lemma 2.1). This is the sequence A094648 in Sloane's Encyclopedia [6]. Next we extend the domain of $\left\{a_{n}\right\}_{n=0}^{\infty}$ to include negative integers. For negative integer $-n$, we have

$$
a_{-n}=\left(4 \cos \frac{\pi}{7} \cos \frac{2 \pi}{7}\right)^{n}+\left(-4 \cos \frac{\pi}{7} \cos \frac{3 \pi}{7}\right)^{n}+\left(4 \cos \frac{2 \pi}{7} \cos \frac{3 \pi}{7}\right)^{n} .
$$

Note that $\left\{a_{n}\right\}_{n=-\infty}^{\infty}$ is an integer sequence, so we can consider this sequence as a sequence in $\mathbb{F}_{p}$. Here we investigate the relation between this sequence in $\mathbb{F}_{p}$ and permutation properties of binomial $P(x)=x^{r}\left(1+x^{e s}\right)$ over a finite field $\mathbb{F}_{q}=\mathbb{F}_{p^{m}}$. We have the following Theorem.

Theorem 1.1. Let $q-1=7 s$ and $1 \leq e \leq 6$. Then $P(x)=x^{r}\left(1+x^{e s}\right)$ is a permutation binomial of $\mathbb{F}_{q}$ if and only if $(r, s)=1,2^{s} \equiv 1(\bmod p), 2 r+e s \equiv \equiv 0(\bmod 7)$, and $\left\{a_{n}\right\}$ satisfies one of the following:

(a) $a_{s}=a_{-s}=3$ in $\mathbb{F}_{p}$;

(b) $a_{-c s-1}=-1+\alpha, a_{-c s}=-1-\alpha$, and $a_{-c s+1}=1$ in $\mathbb{F}_{p}$, where $c$ is the inverse of $s+$ $2 e^{5} r$ modulo 7 and $\alpha^{2}+\alpha+2=0$ in $\mathbb{F}_{p}$.

The sequence $\left\{a_{n}\right\}$ is called $s$-periodic over $\mathbb{F}_{p}$ if $a_{n}=a_{n+k s}$ in $\mathbb{F}_{p}$ for integers $k$ and $n$. Condition (a) in the above theorem is equivalent to $s$-periodicity of $a_{n}$ over $\mathbb{F}_{p}$ (see Lemma 2.4). Equivalently we can say $\left\{a_{n}\right\}$ is $s$-periodic over $\mathbb{F}_{p}$ whenever $\left\{a_{n}\right\}=\left\{a_{n}^{0}\right\}$ in $\mathbb{F}_{p}$, where $\left\{a_{n}^{0}\right\}_{n=-\infty}^{\infty}$ is the unique sequence in $\mathbb{F}_{p}$ defined by the recursion (1.3) and initial values $a_{s-1}^{0}=2, a_{s}^{0}=3$, and $a_{s+1}^{0}=1$. Similarly condition (b) can be written as $\left\{a_{n}\right\}=$ $\left\{a_{n}^{c, \alpha}\right\}$ in $\mathbb{F}_{p}$, where $\left\{a_{n}^{c, \alpha}\right\}_{n=-\infty}^{\infty}$ is the unique sequence in $\mathbb{F}_{p}$ defined by the recursion (1.3) and initial values $a_{-c s-1}=-1+\alpha, a_{-c s}=-1-\alpha$, and $a_{-c s+1}=1$. So Theorem 1.1 states that under certain conditions on $r, s=(q-1) / 7$, and $e$ the binomial $P(x)=x^{r}\left(1+x^{e s}\right)$ is a permutation binomial of $\mathbb{F}_{p}$ if and only if the Lucas-type sequence $\left\{a_{n}\right\}$ is equal to $\left\{a_{n}^{0}\right\}$ or $\left\{a_{n}^{c, \alpha}\right\}$ in $\mathbb{F}_{p}$ (for more explanation, see Example 3.2).

It is clear that if the Legendre symbol $\left(\frac{p}{7}\right)=-1$, then condition (b) in the above theorem is never satisfied (the equation $x^{2}+x+2=0$ does not have any solution in $\mathbb{F}_{p}$ ). Moreover, in this case, we can show that condition (a) is always satisfied, and so we have the following.

Corollary 1.2. Let $q-1=7 s, 1 \leq e \leq 6$, and let $p$ be a prime with $\left(\frac{p}{7}\right)=-1$. Then $P(x)=$ $x^{r}\left(1+x^{e s}\right)$ is a permutation binomial of $\mathbb{F}_{q}$ if and only if $(r, s)=1,2^{s} \equiv 1(\bmod p)$, and $2 r+e s \not \equiv 0(\bmod 7)$. 
Theorem 1.1 gives a complete characterization of permutation binomials of the form $P(x)=x^{r}\left(1+x^{e(q-1) / 7}\right)$. Moreover, our theorem together with the above corollary can lead to an efficient algorithm for constructing such permutation binomials. Note that $\left\{a_{n}\right\}$ is a recursive sequence and therefore conditions (a) and (b) can be quickly verified and so by employing the above theorem it is easy to find new permutation binomials over certain $\mathbb{F}_{q}$. Also by an argument similar to the proof of [1, Corollary 1.3], we can show that under the conditions of Theorem 1.1 on $q$, there are exactly $3 \phi(q-1)$ permutation binomials $P(x)=x^{r}\left(1+x^{e(q-1) / 7}\right)$ of $\mathbb{F}_{q}$. Here, $\phi$ is the Euler totient function.

In the next section, we study certain properties of the sequence $\left\{a_{n}\right\}$ that will be used in the proof of our theorem. Theorem 1.1 and Corollary 1.2 are proved in Section 3.

\section{The sequence $\left\{a_{n}\right\}$}

We first show that $\left\{a_{n}\right\}$ appears in the closed expression for the lacunary sum of binomial coefficients

$$
S(2 n, 7, a):=\sum_{\substack{k=0 \\
k=a(\bmod 7)}}^{2 n}\left(\begin{array}{c}
2 n \\
k
\end{array}\right)
$$

Lemma 2.1. The sequence $\left\{a_{n}\right\}_{n=0}^{\infty}$ satisfies the recursion $a_{n}=a_{n-1}+2 a_{n-2}-a_{n-3}(n \geq 3)$, $a_{0}=3, a_{1}=1, a_{2}=5$, and

$$
S(2 n, 7, a)= \begin{cases}\frac{2^{2 n}+2 a_{2 n}}{7} & \text { if } 2 n-2 a \equiv 0(\bmod 7), \\ \frac{2^{2 n}-a_{2 n+1}}{7} & \text { if } 2 n-2 a \equiv 1,6(\bmod 7), \\ \frac{2^{2 n}+a_{2 n+1}-a_{2 n-1}}{7} & \text { if } 2 n-2 a \equiv 2,5(\bmod 7), \\ \frac{2^{2 n}-a_{2 n}+a_{2 n-1}}{7} & \text { if } 2 n-2 a \equiv 3,4(\bmod 7) .\end{cases}
$$

Proof. Note that $2 \cos (\pi / 7),-2 \cos (2 \pi / 7)$, and $2 \cos (3 \pi / 7)$ are the roots of the polynomial $g(x)=x^{3}-x^{2}-2 x+1$, so $a_{n}$ satisfies the given recursion.

We know that

$$
S(2 n, 7, a)=\frac{2^{2 n}}{7}+\frac{2}{7}\left[\sum_{t=1}^{3}\left(2 \cos \frac{\pi t}{7}\right)^{2 n} \cos \frac{\pi t}{7}(2 n-2 a)\right],
$$

(see [7, page 232, Lemma 1.3]). This together with (1.2) and (1.3) implies the result.

Next we have a general formula for the product $a_{n} a_{m}$.

Lemma 2.2. Let $m$ and $n$ be integers and $m \leq n$. Then

$$
a_{n} a_{m}=a_{m+n}+(-1)^{m}\left(a_{-m} a_{n-m}-a_{n-2 m}\right) .
$$

In particular,

$$
a_{n}^{2}=a_{2 n}+(-1)^{n} 2 a_{-n} .
$$




\section{On some permutation polynomials over finite fields}

Proof. Let $\delta=2 \cos (\pi / 7), \eta=-2 \cos (2 \pi / 7)$, and $\epsilon=2 \cos (3 \pi / 7)$. We have $a_{n}=\delta^{n}+\eta^{n}+$ $\epsilon^{n}$ and $a_{-n}=(-\delta \eta)^{n}+(-\delta \epsilon)^{n}+(-\eta \epsilon)^{n}$. Considering these, a routine calculation implies the result.

In the next two lemmas, we study the periodicity of $\left\{a_{n}\right\}$ over $\mathbb{F}_{p}$.

Lemma 2.3. Let $p \neq 2,7$ be a prime. Then the sequence $\left\{a_{n}\right\}_{n=-\infty}^{\infty}$ is 7 s-periodic over $\mathbb{F}_{p}$.

Proof. We know that $g(x)=x^{3}-x^{2}-2 x+1$ is the characteristic polynomial of the recursion associated to $a_{n}$. Let $\delta, \eta$, and $\epsilon$ be the roots of $g(x)$ in a splitting field $F$ of $g(x)$ over $\mathbb{F}_{p}$. Since $p \neq 2,7$, we know that $a_{n}$ is 7 s-periodic in $\mathbb{F}_{p}$ if and only if $\delta^{7 s}=\eta^{7 s}=\epsilon^{7 s}=1$ in $F$.

We can show that $g(x)$ is either irreducible in $\mathbb{F}_{p}[x]$ or it splits in $\mathbb{F}_{p}[x]$. Now if $g(x)$ splits over $\mathbb{F}_{p}$, then $\delta^{p-1}=\eta^{p-1}=\epsilon^{p-1}=1$ in $\mathbb{F}_{p}$ and therefore $a_{n}$ has period $7 s=q-1$. If $p=7 k+1$ or 6 , by [5, Theorem 7], $g(x)$ splits over $\mathbb{F}_{p}$. If $p=7 k+2,3,4$, or 5 and $g(x)$ is irreducible over $\mathbb{F}_{p}$, then, by [3, Theorems 8.27 and 8.29], $a_{n}$ is periodic in $\mathbb{F}_{p}$ with the least period dividing $p^{3}-1$. Also since $q-1=p^{m}-1 \equiv 0(\bmod 7)$, in these cases, $3 \mid m$. Hence, $a_{n}$ is periodic in $\mathbb{F}_{p}$ with the least period dividing $7 s=q-1$.

We continue by describing a necessary and sufficient condition under which the sequence $\left\{a_{n}\right\}_{n=-\infty}^{\infty}$ will be a periodic sequence in $\mathbb{F}_{p}$ with the even period $s$.

Lemma 2.4. Let $p \neq 2,7$ be a prime and let $s$ be a fixed even positive integer. Then

$$
\left\{a_{n}\right\} \text { is s-periodic over } \mathbb{F}_{p} \Longleftrightarrow a_{s}=a_{-s}=3 \text { in } \mathbb{F}_{p} \text {. }
$$

Proof. With the notation in the proof of Lemma 2.3, we know that $\left\{a_{n}\right\}_{n=-\infty}^{\infty}$ is $s$-periodic if and only if $\operatorname{diag}(\delta, \eta, \epsilon)^{s}=I$ in $F$. Here $\operatorname{diag}(\delta, \eta, \epsilon)$ is a diagonal matrix with entries $\delta$, $\eta$, and $\epsilon$ and $I$ is the identity matrix. We know that a diagonal matrix is equal to the identity matrix if and only if $(x-1)^{3}$ is the characteristic polynomial of the diagonal matrix. By employing this fact, together with the identities $a_{n}=\delta^{n}+\eta^{n}+\epsilon^{n}$ and $a_{-n}=$ $(-\delta \eta)^{n}+(-\delta \epsilon)^{n}+(-\eta \epsilon)^{n}$ in $F$, we have

$$
\operatorname{diag}(\delta, \eta, \epsilon)^{s}=I \text { in } F \Longleftrightarrow a_{s}=a_{-s}=3 \text { in } \mathbb{F}_{p} .
$$

The following two lemmas play important roles in the proof of Theorem 1.1.

Lemma 2.5. Let $p \neq 2,7$ be a prime, $s=(q-1) / 7$, and let $c(1 \leq c \leq 6)$ be a fixed integer. If the sequence $\left\{a_{n}\right\}_{n=-\infty}^{\infty}$ satisfies $a_{c s+1}=a_{2 c s-1}-a_{2 c s+1}=a_{3 c s}-a_{3 c s-1}=a_{4 c s}-a_{4 c s-1}=$ $a_{5 c s-1}-a_{5 c s+1}=a_{6 c s+1}=1$ in $\mathbb{F}_{p}$, then

$$
a_{c s}=a_{2 c s}=a_{4 c s}, \quad a_{3 c s}=a_{5 c s}=a_{6 c s}
$$

in $\mathbb{F}_{p}$.

Proof. From the recurrence relation of $a_{n}$, we get $a_{2 c s-1}-a_{2 c s+1}=2 a_{2 c s}-a_{2 c s+2}$. So, by the conditions of the lemma, we have
(A) $a_{c s+1}^{2}=1$;
(B) $\left(2 a_{2 c s}-a_{2 c s+2}\right)^{2}=1$;
(C) $\left(a_{4 c s}-a_{4 c s-1}\right)^{2}=1$. 
We employ Lemmas 2.2 and 2.3 to deduce new identities from (A), (B), and (C). For simplicity of our exposition, we let $a_{-(c s+1)}=\gamma$.

First of all (A) together with Lemma 2.2 implies

$$
a_{2 c s+2}=1+2 \gamma \text {. }
$$

From (2.9) and $2 a_{2 c s}-a_{2 c s+2}=1$, we have

$$
a_{2 c s}=1+\gamma
$$

Next from (B), (2.9), (2.10), Lemma 2.2, and $a_{c s+1}=1$, we get

$$
\begin{aligned}
1 & =\left(2 a_{2 c s}-a_{2 c s+2}\right)^{2} \\
& =4 a_{2 c s}^{2}-4 a_{2 c s} a_{2 c s+2}+a_{2 c s+2}^{2} \\
& =-4(1+\gamma) \gamma+a_{2 c s+2}^{2} \\
& =-4(1+\gamma) \gamma+a_{4 c s+4}+2 a_{-(2 c s+2)} \\
& =-4(1+\gamma) \gamma+a_{4 c s+4}+2\left(\gamma^{2}+2\right) .
\end{aligned}
$$

This implies

$$
a_{4 c s+4}=2(1+\gamma)^{2}-5=2 a_{2 c s}^{2}-5 .
$$

Note that $a_{4 c s}-a_{4 c s-1}=1$ and the recurrence relation (1.3) imply

$$
a_{4 c s+2}=a_{4 c s+1}+a_{4 c s}+1
$$

and

$$
a_{4 c s+3}=3 a_{4 c s+1}+1
$$

Now applying the recurrence relation $a_{4 c s+4}=a_{4 c s+3}+2 a_{4 c s+2}-a_{4 c s+1}$ together with (2.13) and (2.14) to the left-hand side of (2.12) and applying Lemmas 2.2 and 2.3 to the right-hand side of (2.12) yield

$$
a_{4 c s+1}=a_{5 c s}-2 .
$$

Finally, from $(\mathrm{C})$, we have

$$
a_{4 c s}^{2}-2 a_{4 c s} a_{4 c s-1}+a_{4 c s-1}^{2}=1 .
$$

Applying Lemmas 2.2 and 2.3 on this equality yields

$$
a_{c s}+2 a_{3 c s}-2 a_{c s-1}-2 a_{3 c s+2}+a_{c s-2}=1 .
$$

Now by employing the recurrence relation $a_{c s+1}=a_{c s}+2 a_{c s-1}-a_{c s-2}$ in the previous identity and $a_{c s+1}=1$, we obtain

$$
a_{c s}=a_{3 c s+2}-a_{3 c s}+1 .
$$


2636 On some permutation polynomials over finite fields

Since $a_{3 c s}-a_{3 c s-1}=1$, from the recurrence relation (1.3), we have

$$
a_{3 c s+2}=a_{3 c s+1}+a_{3 c s}+1 .
$$

Applying this identity in (2.18) yields

$$
a_{c s}=a_{3 c s+1}+2 \text {. }
$$

Now we are ready to finish the proof. Note that by changing $s$ to $-s$ all the above equations remain true, so, by changing $s$ to $-s$ in (2.15) and applying Lemma 2.3, we have

$$
a_{3 c s+1}=a_{2 c s}-2 .
$$

This together with (2.20) implies $a_{c s}=a_{2 c s}$. Changing $s$ to $-s$ in this equality yields $a_{6 c s}=a_{5 c s}$. These identities together with Lemmas 2.2 and 2.3 imply that

$$
a_{c s}=a_{2 c s}=a_{4 c s}, \quad a_{3 c s}=a_{5 c s}=a_{6 c s} .
$$

LemMA 2.6. Let $p \neq 2,7$ be a prime, $s=(q-1) / 7$, and let $c(1 \leq c \leq 6)$ be a fixed integer. If the sequence $\left\{a_{n}\right\}_{n=-\infty}^{\infty}$ satisfies

$$
a_{6 c s-1}=-1+\alpha, \quad a_{6 c s}=-1-\alpha, \quad a_{6 c s+1}=1,
$$

where $\alpha$ is a root of equation $x^{2}+x+2=0$ in $\mathbb{F}_{p}$, then we have $a_{c s}=a_{2 c s}=a_{4 c s}=\alpha, a_{3 c s}=$ $a_{5 c s}=a_{6 c s}=-1-\alpha, a_{c s-1}=-2-\alpha, a_{c s+1}=1, a_{5 c s-1}=1-2 \alpha$, and $a_{5 c s+1}=-2 \alpha$ in $\mathbb{F}_{p}$.

Proof. From Lemmas 2.2 and 2.3, we have the following six identities:

$$
\begin{gathered}
a_{6 c s-1}^{2}=a_{5 c s-2}-2 a_{c s+1}, \\
a_{6 c s-1} a_{6 c s}=a_{5 c s-1}-a_{1} a_{c s+1}+a_{c s+2}, \\
a_{6 c s-1} a_{6 c s+1}=a_{5 c s}-a_{2} a_{c s+1}+a_{c s+3}, \\
a_{6 c s}^{2}=a_{5 c s}+2 a_{c s}, \\
a_{6 c s} a_{6 c s+1}=a_{5 c s+1}+a_{c s}-a_{c s+1}, \\
a_{6 c s+1}^{2}=a_{5 c s+2}-2 a_{c s-1} .
\end{gathered}
$$

Replacing the known values of the variables in the above identities, writing $a_{5 c s-2}$ and $a_{5 c s+2}$ in terms of $a_{5 c s-1}, a_{5 c s}$, and $a_{5 c s+1}$, and writing $a_{c s+2}$ and $a_{c s+3}$ in terms of $a_{c s-1}, a_{c s}$, and $a_{c s+1}$ yield

$$
\begin{gathered}
(-1+\alpha)^{2}=2 a_{5 c s-1}+a_{5 c s}-a_{5 c s+1}-2 a_{c s+1}, \\
1-\alpha^{2}=a_{5 c s-1}-a_{c s-1}+2 a_{c s}, \\
-1+\alpha=a_{5 c s}-a_{c s-1}+a_{c s}-2 a_{c s+1}, \\
(1+\alpha)^{2}=a_{5 c s}+2 a_{c s}, \\
-1-\alpha=a_{5 c s+1}+a_{c s}-a_{c s+1} \\
1=-a_{5 c s-1}+2 a_{5 c s}+a_{5 c s+1}-2 a_{c s-1} .
\end{gathered}
$$


Solving this system of linear equations and noting that $\alpha^{2}+\alpha+2=0$ imply the desired values for $a_{c s-1}, a_{c s}, a_{c s+1}, a_{5 c s-1}, a_{5 c s}$, and $a_{5 c s+1}$. By setting up two similar systems of linear equations, one can derive the desired values for $a_{2 c s}, a_{3 c s}$, and $a_{4 c s}$.

\section{Permutation binomials and the sequence $\left\{a_{n}\right\}$}

The main tool in the proof of Theorem 1.1 is the following well-known theorem of Hermite [3, Theorem 7.4].

Theorem 3.1 (Hermite's criterion). $P(x)$ is a permutation polynomial of $\mathbb{F}_{q}$ if and only if

(i) $P(x)$ has exactly one root in $\mathbb{F}_{q}$;

(ii) for each integer $t$ with $1 \leq t \leq q-2$ and $t \neq \equiv 0(\bmod p)$, the reduction of $[P(x)]^{t}$ $\bmod \left(x^{q}-x\right)$ has degree less than or equal to $q-2$.

Finally, we are ready to prove the main result of this paper.

Proof of Theorem 1.1. First we assume that $P(x)$ is a permutation binomial. Then $p \neq 2$, since otherwise $P(0)=P(1)=0$. Also, in this case, it is known that $(r, s)=1[8$, Theorem 1.2] and $2^{s} \equiv 1(\bmod p)\left[4\right.$, Theorem 4.7]. Next we note that the coefficient of $x^{q-1}$ in the expansion of $[P(x)]^{k s}$ is $S\left(k s, 7,-k e^{5} r\right)$, so if $P(x)$ is a permutation binomial, then by Hermite's criterion $S\left(k s, 7,-k e^{5} r\right)=0$ in $\mathbb{F}_{p}$ for $k=1, \ldots, 6$.

We next show that $2 r+e s \not \equiv 0(\bmod 7)$. Otherwise, $2 r+e s \equiv 0(\bmod 7)$ and Lemma 2.1 yields that

$$
S\left(k s, 7,-k e^{5} r\right)=\frac{2^{k s}+2 a_{k s}}{7} \text { in } \mathbb{F}_{p}
$$

for $k=1, \ldots, 6$. From here if $P(x)$ is a permutation binomial, we have

$$
a_{s}=a_{2 s}=\cdots=a_{6 s}=-\frac{1}{2} \text { in } \mathbb{F}_{p}
$$

Using Lemmas 2.3 and 2.2, we have $1 / 4=a_{s}^{2}=a_{2 s}+2 a_{6 s}=3 a_{s}=-3 / 2$. Hence, $(1 / 2)$ $((1 / 2)+3)=0$ in $\mathbb{F}_{p}$ which is a contradiction since $7 \mid(q-1)$. Hence, $2 r+e s \not \equiv 0(\bmod 7)$.

It remains to show that if $P(x)$ is a permutation binomial, then either (a) or (b) holds. Let $c$ be the inverse of $s+2 e^{5} r$ modulo7. Hermite's criterion together with Lemma 2.1 implies that

$$
\begin{aligned}
& a_{c s+1}=1, \quad a_{2 c s-1}-a_{2 c s+1}=1, \quad a_{3 c s}-a_{3 c s-1}=1, \\
& a_{4 c s}-a_{4 c s-1}=1, \quad a_{5 c s-1}-a_{5 c s+1}=1, \quad a_{6 c s+1}=1,
\end{aligned}
$$

in $\mathbb{F}_{p}$. So, by Lemma 2.5 , we have

$$
a_{c s}=a_{2 c s}=a_{4 c s}=\alpha, \quad a_{3 c s}=a_{5 c s}=a_{6 c s}=\beta,
$$

in $\mathbb{F}_{p}$. From Lemmas 2.2 and 2.3 , we have

$$
a_{c s}^{2}=a_{2 c s}+2 a_{6 c s}, \quad a_{6 c s}^{2}=a_{5 c s}+2 a_{c s} .
$$


2638 On some permutation polynomials over finite fields

By subtracting these two equations and employing (3.4), we get

$$
\left(a_{c s}-a_{6 c s}\right)\left(a_{c s}+a_{6 c s}+1\right)=0 \text { in } \mathbb{F}_{p}
$$

If $\alpha=\beta$ in $\mathbb{F}_{p}$, then by Lemma 2.2 and (3.4) we have $a_{7 c s}=a_{c s} a_{6 c s}-a_{6 c s} a_{5 c s}+a_{4 c s}=a_{4 c s}$. Since by Lemma $2.3 a_{7 c s}=a_{0}=3$ in $\mathbb{F}_{p}$, we have $a_{4 c s}=3$ in $\mathbb{F}_{p}$. This together with (3.4) and $a_{c s}=a_{6 c s}$ implies condition (a).

If $\alpha \neq \beta$, then from (3.6) we have $a_{c s}+a_{6 c s}+1=0$. This together with (3.5) implies that $\alpha$ and $\beta$ are roots of the equation $x^{2}+x+2=0$ in $\mathbb{F}_{p}$ and therefore $\beta=-1-\alpha$.

From Lemma 2.2, we have

$$
a_{c s} a_{c s+1}=a_{2 c s+1}+a_{6 c s} a_{1}-a_{6 c s+1}
$$

This together with $a_{c s}=\alpha, a_{6 c s}=-1-\alpha$, and $a_{c s+1}=a_{6 c s+1}=1$ implies that $a_{2 c s+1}=2 \alpha+$ 2. Note that $a_{2 c s-1}=1+a_{2 c s+1}$, and so $a_{2 c s-1}=2 \alpha+3$ and thus $a_{2 c s+2}=a_{2 c s+1}+2 a_{2 c s}-$ $a_{2 c s-1}=2 \alpha-1$. Finally, by Lemma 2.2, we have $a_{c s+1}^{2}=a_{2 c s+2}-2 a_{6 c s-1}$ which implies $a_{6 c s-1}=\alpha-1$. Hence, in this case, $a_{n}$ satisfies condition (b).

Conversely we assume that the conditions in Theorem 1.1 are satisfied and we show that $P(x)$ is a permutation binomial. First note that $2^{s} \equiv 1(\bmod p)$ follows that $p$ is odd. Hence, it is obvious that $P(x)$ has only one root in $\mathbb{F}_{q}$. Since, $(r, s)=1$, the possible coefficient of $x^{q-1}$ in the expansion of $[P(x)]^{t}$ can only happen if $t=k s$ for some $k=1, \ldots, 6$. So by Hermite's criterion, it is sufficient to show that $S\left(k s, 7,-k e^{5} r\right)=0$ in $\mathbb{F}_{p}$ for $k=1, \ldots, 6$.

Now if $a_{n}$ satisfies condition (a), then by Lemma $2.4 a_{n}$ is $s$-periodic over $\mathbb{F}_{p}$. Using the initial values of $a_{n}, 2 r+e s \not \equiv 0(\bmod 7)$, and Lemma 2.1 , we have $S\left(k s, 7,-k e^{5} r\right)=0$ in $\mathbb{F}_{p}$ and thus $P(x)$ is a permutation binomial over $\mathbb{F}_{q}$.

Next we assume that $a_{n}$ satisfies condition (b). Then, by Lemma 2.6, we also have

$$
\begin{gathered}
a_{c s}=a_{2 c s}=a_{4 c s}=\alpha, \quad a_{3 c s}=a_{5 c s}=a_{6 c s}=-1-\alpha, \\
a_{c s-1}=-2-\alpha, \quad a_{c s+1}=1, \quad a_{5 c s-1}=1-2 \alpha, \quad a_{5 c s+1}=-2 \alpha .
\end{gathered}
$$

By using $2^{s}=1, a_{c s+1}=a_{6 c s+1}=1$, and Lemma 2.1, we have

$$
S\left(k c s, 7,-k c e^{5} r\right)=0 \quad \text { for } k=1,6
$$

To demonstrate $S\left(k c s, 7,-k c e^{5} r\right)=0$ for other $k$ 's, it is sufficient to show that

$$
\begin{aligned}
& a_{2 c s-1}-a_{2 c s+1}=1, \quad a_{3 c s}-a_{3 c s-1}=1, \\
& a_{4 c s}-a_{4 c s-1}=1, \quad a_{5 c s-1}-a_{5 c s+1}=1 .
\end{aligned}
$$

From the values for $a_{5 c s-1}$ and $a_{5 c s+1}$, it is clear that $a_{5 c s-1}-a_{5 c s+1}=1$. Next note that by considering appropriate systems of linear equations as described in the proof of Lemma 2.6, we can deduce that

$$
a_{2 c s-1}=2 \alpha+3, \quad a_{2 c s+1}=2 \alpha+2, \quad a_{3 c s-1}=-\alpha-2, \quad a_{4 c s-1}=\alpha-1 .
$$


Table 3.1

\begin{tabular}{cccc}
\hline Type IV & Type III & Type II & Type I \\
\hline 2731 & 4999 & 7309 & 874651 \\
3389 & 18439 & 20063 & 941879 \\
15583 & 20441 & 33587 & 1018879 \\
62791 & 33503 & 37199 & 1036267 \\
65899 & 55609 & 37339 & 1074277 \\
$\vdots$ & $\vdots$ & $\vdots$ & $\vdots$ \\
\hline
\end{tabular}

So $a_{2 c s-1}-a_{2 c s+1}=a_{3 c s}-a_{3 c s-1}=a_{4 c s}-a_{4 c s-1}=1$. These relations show that $S(k s, 7$, $\left.-k e^{5} r\right)=0$ in $\mathbb{F}_{p}$ for $k=1, \ldots, 6$. Hence, $P(x)$ is a permutation binomial of $\mathbb{F}_{q}$.

Next we prove that if $\left(\frac{p}{7}\right)=-1$ then the sequence $a_{n}$ is always $s$-periodic. That is, $a_{s}=a_{-s}=3$.

Proof of Corollary 1.2. Following the notation in the proof of Lemma 2.3, let $\epsilon$ be a root of $g(x)=x^{3}-x^{2}-2 x+1$ in an extension of $\mathbb{F}_{p}$. We need to prove that $\epsilon^{s}=1$. If $p \equiv$ $6(\bmod 7)$, then by $\left[5\right.$, Theorem 7] we have $\epsilon \in \mathbb{F}_{p}$. Since $(p-1,7)=1$, in this case, $\epsilon$ is a 7 th power in $\mathbb{F}_{p}$ and therefore $\epsilon^{s}=1$ in $\mathbb{F}_{p}$. To prove the result for $p \equiv 3$ or $5(\bmod 7)$, first of all note that $g(x)$ is either irreducible in $\mathbb{F}_{p}[x]$ or it splits in $\mathbb{F}_{p}[x]$. If it splits over $\mathbb{F}_{p}$, then $\epsilon$ is a 7 th power in $\mathbb{F}_{p}$ and so $\epsilon^{s}=1$ in $\mathbb{F}_{p}$. Otherwise, $g(x)$ splits over $\mathbb{F}_{p^{3}}$. Now since $p \not \equiv 1,2$ or $4(\bmod 7)$, we have $\left(p^{3}-1,7\right)=1$, so $\epsilon$ is a 7 th power in $\mathbb{F}_{p^{3}}$ and therefore $\epsilon^{\left(p^{3}-1\right) / 7}=1$ in $\mathbb{F}_{p^{3}}$. Also since $7 \mid(q-1)$, we have $6 \mid m$. This and $\epsilon^{\left(p^{3}-1\right) / 7}=1$ in $\mathbb{F}_{p^{3}}$ implies that $\epsilon^{s}=1$ in $\mathbb{F}_{q}$. Hence, $\left\{a_{n}\right\}$ is $s$-periodic and so by Lemma 2.4, $a_{s}=a_{-s}=3$. Now Theorem 1.1 implies the result.

Example 3.2. An algorithm for finding permutation binomials $P(x)=x^{r}\left(1+x^{e(q-1) / 7}\right)$ of a given field $\mathbb{F}_{q}$ can be easily implemented by using Theorem 1.1 and Corollary 1.2. Moreover, our theorem together with Lemmas 2.4 and 2.6 implies that under certain conditions on $r, s$, and $e$ the binomial $x^{r}\left(1+x^{e s}\right)$ is a permutation polynomial over $\mathbb{F}_{q}$ if and only if the Lucas-type sequence $\left\{a_{n}\right\}$ becomes one of the following four sequences over $\mathbb{F}_{p}$ :

(I) $a_{-s-1}=2, a_{-s}=3, a_{-s+1}=1, a_{s-1}=2, a_{s}=3$, and $a_{s+1}=1$;

(II) $a_{-s-1}=-1+\alpha, a_{-s}=-1-\alpha, a_{-s+1}=1, a_{s-1}=-2-\alpha, a_{s}=\alpha$, and $a_{s+1}=1$;

(III) $a_{-2 s-1}=-1+\alpha, a_{-2 s}=-1-\alpha, a_{-2 s+1}=1, a_{2 s-1}=-2-\alpha, a_{2 s}=\alpha$, and $a_{2 s+1}=1$;

(IV) $a_{-3 s-1}=-1+\alpha, a_{-3 s}=-1-\alpha, a_{-3 s+1}=1, a_{3 s-1}=-2-\alpha, a_{3 s}=\alpha$, and $a_{3 s+1}=1$.

Note that the sequence (I) is $s$-periodic and in (II), (III), and (IV), $\alpha$ is a root of equation $x^{2}+x+2=0$ in $\mathbb{F}_{p}$.

Table 3.1 gives some prime numbers $p$ with $p \equiv 1(\bmod 7)$ and $2^{(p-1) / 7} \equiv 1(\bmod p)$ whose corresponding sequence $\left\{a_{n}\right\}$ over $\mathbb{F}_{p}$ is in the form (I) (resp., (II), (III), (IV)).

Here $p=2731$ (resp., 4999, 7309, 874651) is the smallest prime $p \equiv 1(\bmod 7)$ with $2^{(p-1) / 7} \equiv 1(\bmod p)$ whose corresponding sequence $\left\{a_{n}\right\}$ over $\mathbb{F}_{p}$ is in the form (IV) (resp., (III), (II), (I)). Table 3.2 gives examples of such permutation binomials over these four fields. 
2640 On some permutation polynomials over finite fields

Table 3.2

\begin{tabular}{c|cccc}
\hline & $p=2731$ & $p=4999$ & $p=7309$ & $p=874651$ \\
\hline$a_{n}$ & $a_{-3 s-1}=1001$ & $a_{-2 s-1}=760$ & $a_{-s-1}=3858$ & $a_{-s-1}=2$ \\
& $a_{-3 s}=1728$ & $a_{-2 s}=4237$ & $a_{-s}=3449$ & $a_{-s}=3$ \\
& $a_{-3 s+1}=1$ & $a_{-2 s+1}=1$ & $a_{-s+1}=1$ & $a_{-s+1}=1$ \\
& $a_{3 s-1}=1727$ & $a_{2 s-1}=4236$ & $a_{s-1}=3448$ & $a_{s-1}=2$ \\
& $a_{3 s}=1002$ & $a_{2 s}=761$ & $a_{s}=3859$ & $a_{s}=3$ \\
& $a_{3 s+1}=1$ & $a_{2 s+1}=1$ & $a_{s+1}=1$ & $a_{s+1}=1$ \\
\hline$(r, e, s)$ & $(7,1,390)$ & $(5,1,714)$ & $(7,1,1044)$ & $(1,1,124950)$ \\
& $(23,1,390)$ & $(19,1,714)$ & $(13,1,1044)$ & $(11,1,124950)$ \\
& $(37,1,390)$ & $(23,1,714)$ & $(35,1,1044)$ & $(13,1,124950)$ \\
& $(49,1,390)$ & $(37,1,714)$ & $(41,1,1044)$ & $(19,1,124950)$ \\
& $(77,1,390)$ & $(47,1,714)$ & $(49,1,1044)$ & $(23,1,124950)$ \\
& $\vdots$ & $\vdots$ & $\vdots$ & $\vdots$ \\
& & & &
\end{tabular}

\section{Acknowledgments}

Research of both authors was partially supported by NSERC.

\section{References}

[1] A. Akbary and Q. Wang, A generalized Lucas sequence and permutation binomials, Proc. Amer. Math. Soc. 134 (2006), no. 1, 15-22.

[2] J. B. Lee and Y. H. Park, Some permuting trinomials over finite fields, Acta Math. Sci. (English Ed.) 17 (1997), no. 3, 250-254.

[3] R. Lidl and H. Niederreiter, Finite Fields, Encyclopedia of Mathematics and Its Applications, vol. 20, Cambridge University Press, Cambridge, 1997.

[4] Y. H. Park and J. B. Lee, Permutation polynomials with exponents in an arithmetic progression, Bull. Austral. Math. Soc. 57 (1998), no. 2, 243-252.

[5] M. O. Rayes, V. Trevisan, and P. Wang, Factorization of Chebyshev polynomials, http://icm.mcs.kent.edu/reports/index1998.html.

[6] N. J. A. Sloane, The On-Line Encyclopedia of Integer Sequences, http://www.research.att.com/ $\sim$ njas/sequences/.

[7] Z. H. Sun, The combinatorial sum $\sum_{k=0, k \equiv r(\bmod m)}^{n}\left(\begin{array}{l}n \\ k\end{array}\right)$ and its applications in number theory. I, Nanjing Daxue Xuebao Shuxue Bannian Kan 9 (1992), no. 2, 227-240 (Chinese).

[8] D. Q. Wan and R. Lidl, Permutation polynomials of the form $x^{r} f\left(x^{(q-1) / d}\right)$ and their group structure, Monatsh. Math. 112 (1991), no. 2, 149-163.

[9] L. Wang, On permutation polynomials, Finite Fields Appl. 8 (2002), no. 3, 311-322.

Amir Akbary: Department of Mathematics and Computer Science, University of Lethbridge, 4401 University Drive West, Lethbridge, AB, Canada T1K 3M4

E-mail address: akbary@cs.uleth.ca

Qiang Wang: School of Mathematics and Statistics, Carleton University, Ottawa, ON, Canada K1S 5B6

E-mail address: wang@math.carleton.ca 